Supporting Information

\title{
Simultaneous Transfer and Imaging of Latent Fingerprints Enabled by Interfacial Separation of Polydopamine Thin Film
}

Lei Zhao ${ }^{\dagger}$, Wei Wang ${ }^{\ddagger}$, and Weihua $\mathrm{Hu}^{\dagger *}$

$\dagger$ Institute for Clean Energy \& Advanced Materials, Faculty of Materials and Energy; Chongqing Key Laboratory for Advanced Materials and Technologies of Clean Energies, Southwest University, Chongqing 400715, China;

† Singapore Institute of Manufacturing Technology, Agency for Science, Technology and Research (A*STAR) 71 Nanyang Drive, Singapore 638075 


\section{Chemicals and Materials}

Tris (hydroxymethyl) aminomethane, dopamine hydrochloride, silver nitrate $\left(\mathrm{AgNO}_{3}\right)$ were obtained from Sigma-Aldrich. Ammonium persulphate were purchased from Aladdin (China). All chemicals were of analytical grade and used without further purification. Deionized (DI) water (18.2 M $\Omega$ ) was used in all experiments.

\section{Characterization}

SEM images were obtained from a JSM-7800F or JSM-6510LV (JEOL, Japan). UV-Vis spectroscopy was performed with a UV-2550 system (SHIMADZU, Japan). XRD patterns were collected on a SHIMADZU XRD-7000 diffractometer. Raman spectra were gethered on a Renishaw Raman microscopy (Invia Reflex). FTIR spectra were recorded on a Nicolet FTIR 6700 spectrophotometer (Thermo Nicolet) under ATR mode. Optical profilometry images and height histograms were obtained with a Zeta-20 optical profiler from Zeta Instruments, San Jose, CA.

\section{Growing PDA film on PDMS}

PDMS flake with approximate thickness of $1 \mathrm{~mm}$ was prepared by mixing $3 \mathrm{~g}$ elastomeric base and $0.3 \mathrm{~g}$ curing agent in a nine-millimeter-diameter petri dish and cured at $70^{\circ} \mathrm{C}$ for $2 \mathrm{~h}$. Then the PDMS was cut into small pieces and immersed in a freshly prepared dopamine solution $\left(2.0 \mathrm{mg} \mathrm{mL}{ }^{-1}\right.$ dopamine and $2.0 \mathrm{mg} \mathrm{mL}{ }^{-1}$ ammonium persulphate in $50 \mathrm{mM}$ Tris buffer, $\mathrm{pH} 8.5)^{[1]}$ under ambient atmosphere for $2 \mathrm{~h}$. The immersion-growth process was repeated twice. After that, the PDMS with coated PDA thin film was rinsed with copious deionized water and dried with gentle $\mathrm{N}_{2}$ flow for subsequent use.

\section{Fingerprints collection}

Fingermarks enhanced with the forehead sebum were laid directly on a chosen surface that were pre-cleaned with water and ethanol. Before the Cover-separation process, the substrate carrying LFP was heated at $60{ }^{\circ} \mathrm{C}$ for $5 \mathrm{~min}$ or gently flowed with hot air (ca. $\left.60{ }^{\circ} \mathrm{C}\right)$ for $1 \mathrm{~min}$.

\section{Electroless deposition of silver}

Electroless deposition of silver was carried out according to Formanek's report ${ }^{[2]}$. Aqueous ammonia solution $(0.2 \mathrm{M})$ was added dropwise to an aqueous silver nitrate 
solution $(5 \mathrm{mM})$ until brown precipitate was generated and then dissolved. Glucose solution $(1.67 \mathrm{mM})$ was added into the silver nitrate solution $(\mathrm{V} / \mathrm{V}=1: 1)$. The PDA-coated PDMS or PDA-adsorbed substrate was immediately immersed in the solution. After $5 \mathrm{~min}$, the substrate was removed from the solution and rinsed with DI water, and dried with $\mathrm{N}_{2}$.

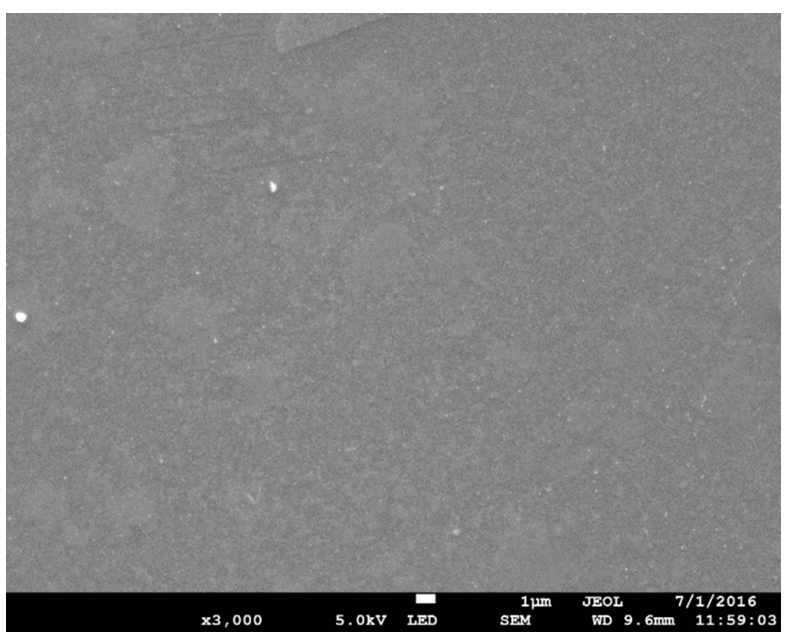

Figure S1. SEM image of groove area of an imaged LFP on glass. No AgNPs or PDA film could be found.

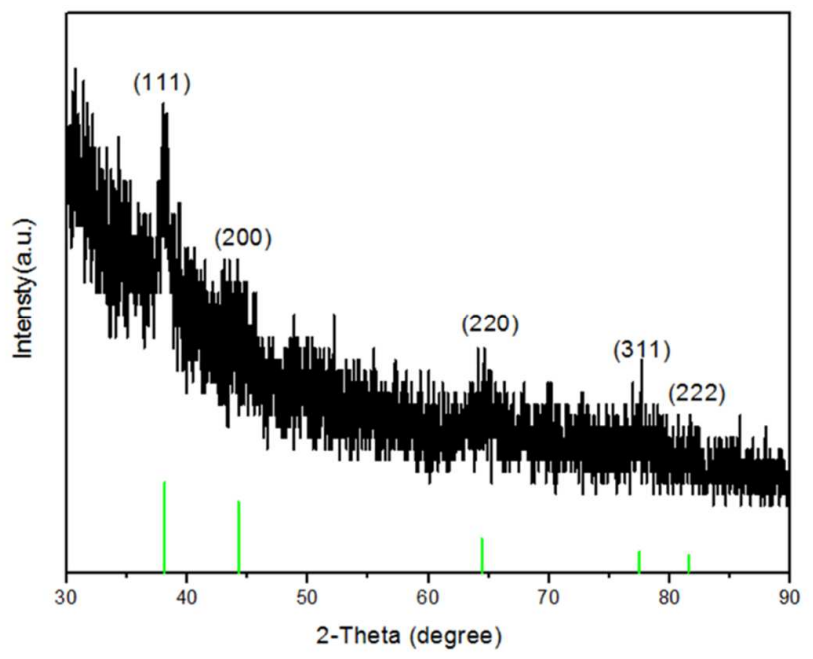

Figure S2. XRD pattern of AgNPs deposited on PDA-coated PDMS 

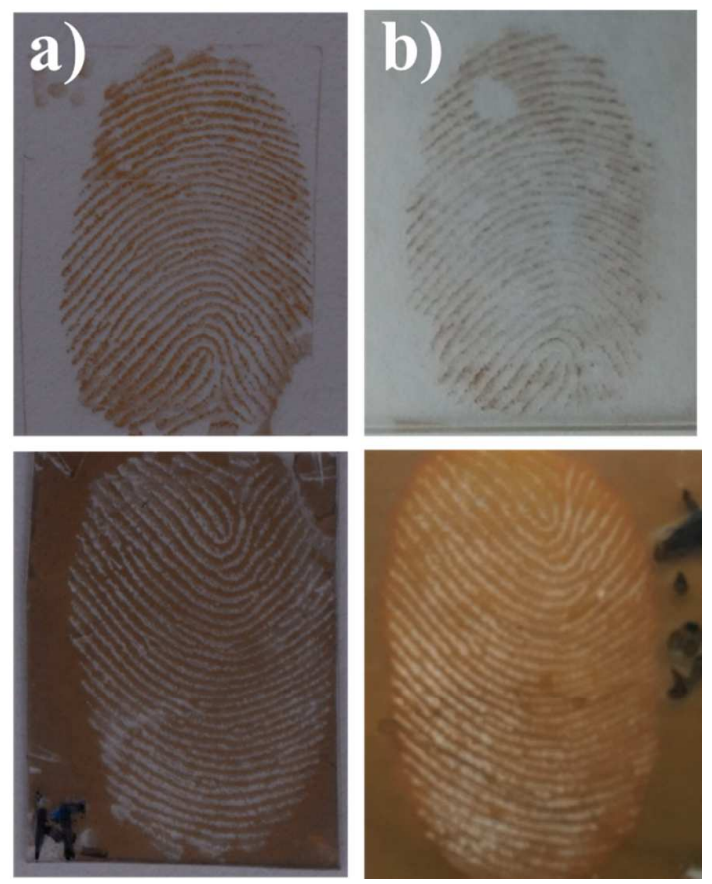

Figure S3. Imaged LFPs on glass (upper row) and corresponding negative images on PDMS (bottom row) after aging for 7 days (a) and 50 days (b).

\section{Fingerprint \\ on glass}

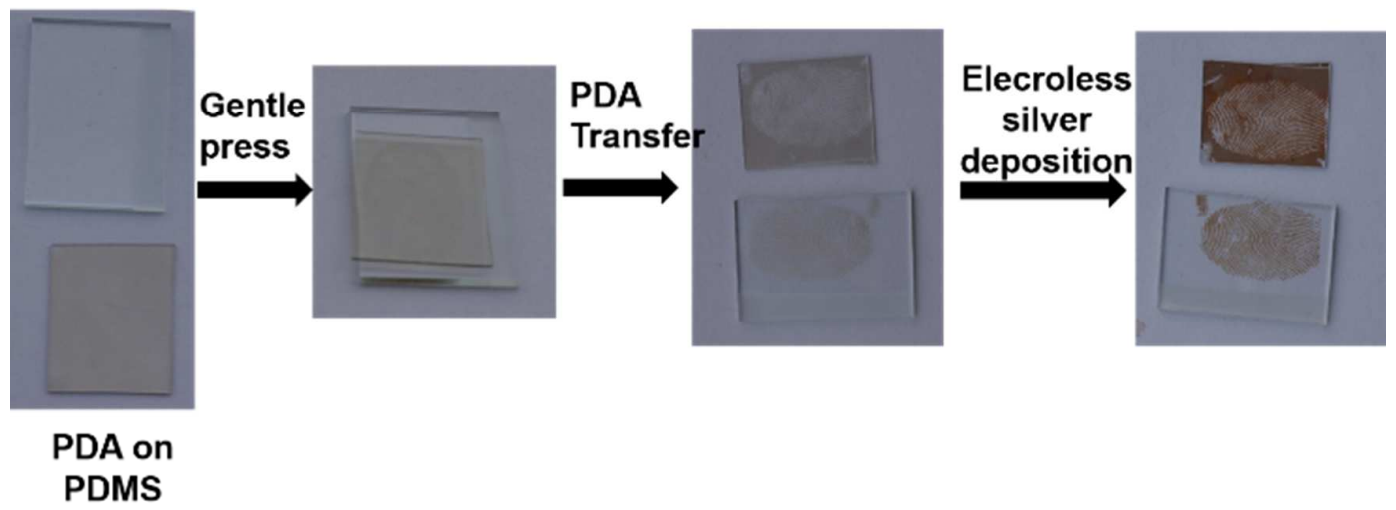

Figure S4. Optical photographs depicting the change of glass with LFP and PDA-coated PDMS flake during cover-separation process. 

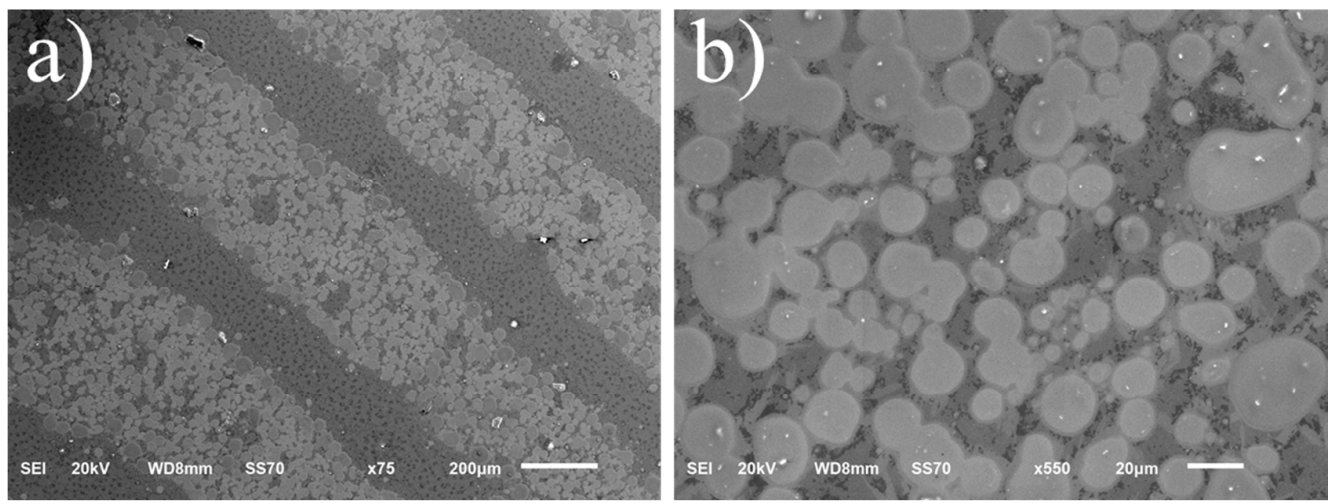

Figure S5. SEM images of a LPF on glass (a) and magnified ridge area (b).

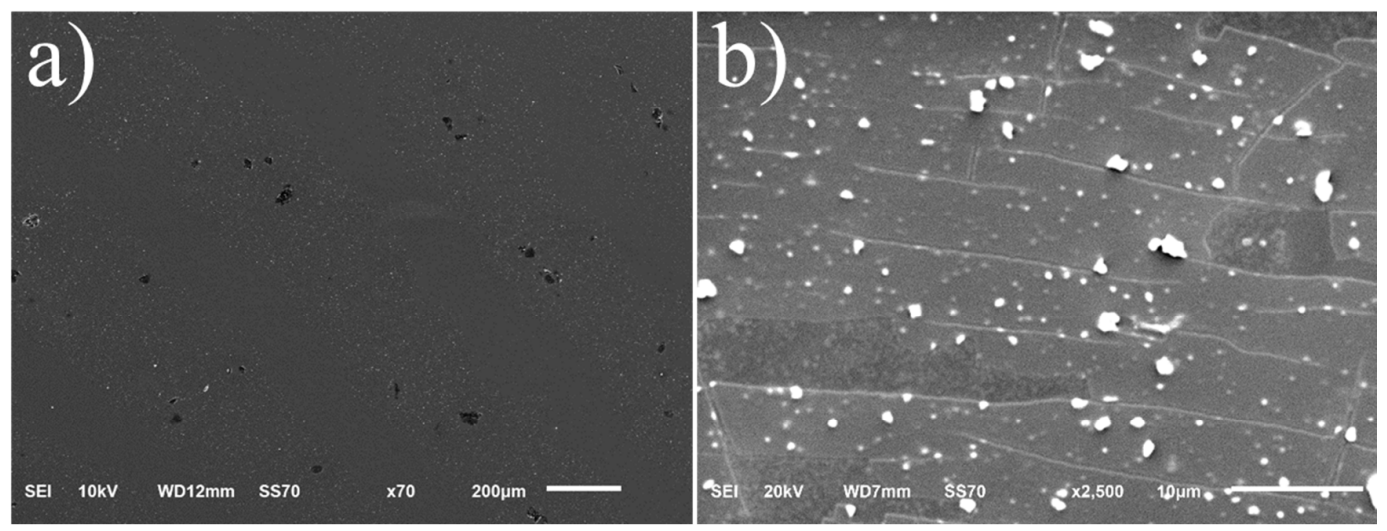

Figure S6. SEM images of a LPF on glass (a) and magnified ridge area (b) after PDA transfer.

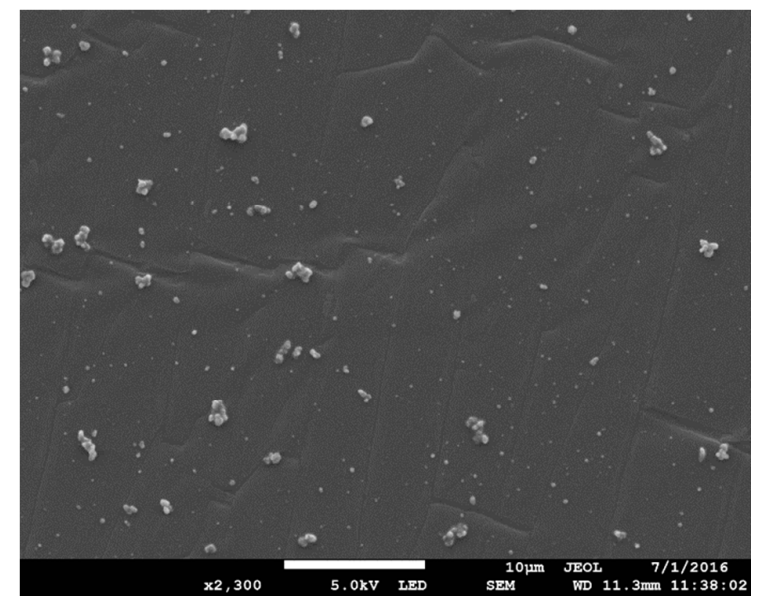

Figure S7. SEM image of PDA-coated PDMS. 

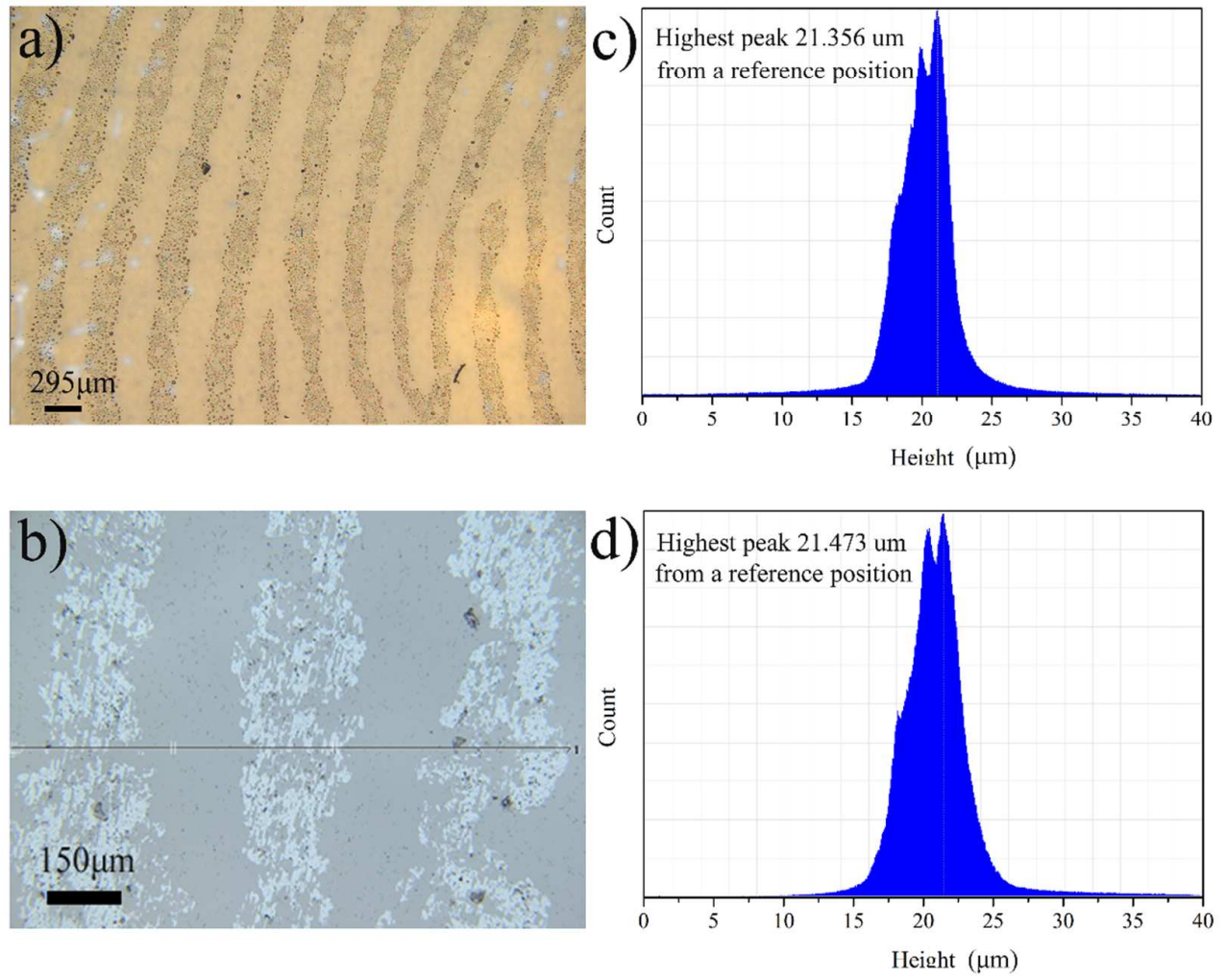

Figure S8. Optical profilometry image (a, 5×; b, 20×) and height histograms of a LFP on glass before (c) and after (d) the cover-separation process.

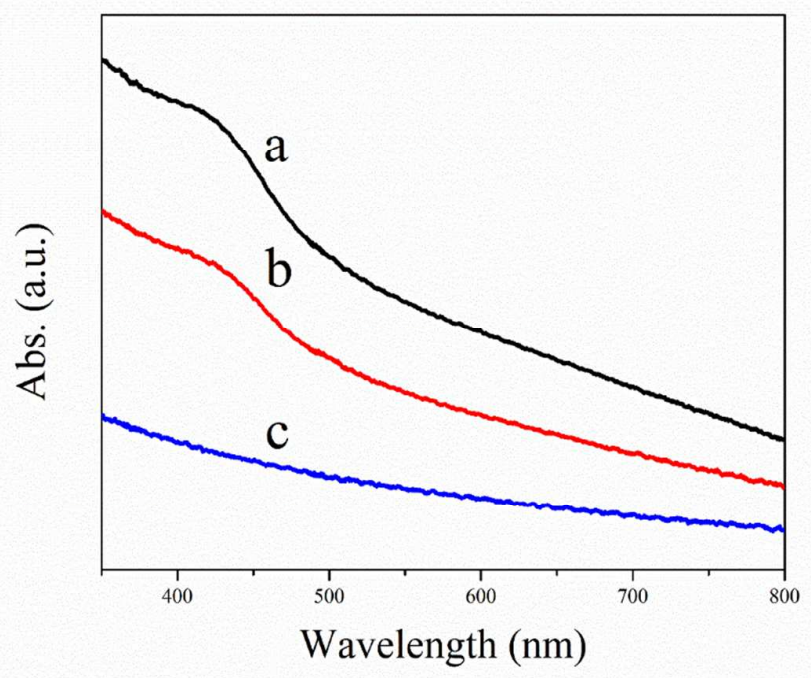

Figure S9. UV-Vis spectra of PDA-coated PDMS (a) and sweat-printed glass before (c) and after PDA transfer (b). 


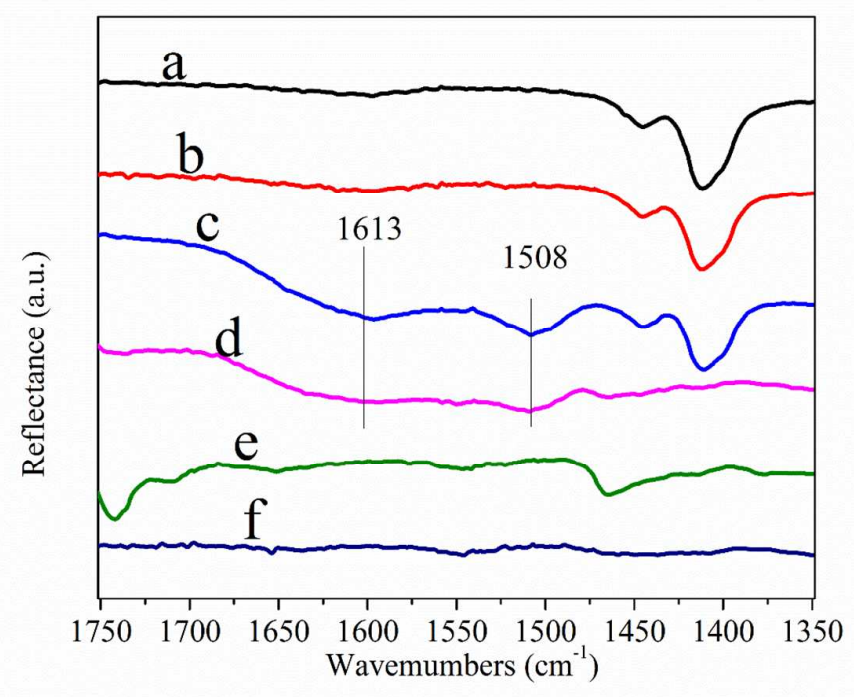

Figure S10. FTIR spectra of PDMS (a); PTFE (f); PDA-coated PDMS before (c) and after PDA-transfer (b); sweat-adsorbed PTFE before (e) and after PDA-transfer (d). After PDA transfer, the characteristic peaks for PDA disappear from PDMS surface but appear on PTFE surface, suggesting the successful PDA transfer from PDMS to PTFE. 


\section{REFERENCES}

1. Wei, Q., Zhang, F., Li, J., Li, B., \& Zhao, C. (2010). Oxidant-induced dopamine polymerization for multifunctional coatings. Polymer Chemistry, 1(9), 1430-1433.

2. Formanek, F.; Takeyasu, N.; Tanaka, T.; Chiyoda, K.; Ishikawa, A.; Kawata, S. Selective electroless plating to fabricate complex three-dimensional metallic micro/nanostructures. Appl. Phys. Lett. 2006, 88 . 